\title{
EL APRENDIZAJE DEL MAPA MENTAL GRUPAL MEDIANTE LAS TIC EN EDUCACIÓN SUPERIOR
}

\author{
Juan Manuel Muñoz González ${ }^{1}$ \\ Esther María Vega GeA² \\ María Dolores Hidalgo Ariza ${ }^{3}$
}

\begin{abstract}
RESUMEN: El objetivo del presente estudio se centra en el aprendizaje del mapa mental como técnica de estudio en los futuros profesionales de la educación a través de una dinámica de aprendizaje cooperativo, así como del software Mindmanager 7 para la construcción del mismo. Para eso, hemos adoptado una metodología cuantitativa, a través del uso de un cuestionario creado ad hoc y conformado por 22 ítems. Los resultados obtenidos ponen de manifiesto que la combinación del uso de las tecnologías de la información y la comunicación (TIC) y del trabajo en equipo contribuye positivamente en el aprendizaje de los mapas mentales, así como en la valoración de estos en el ámbito de estudio, pese a que, en determinados aspectos, la postura del alumnado sea de indiferencia.
\end{abstract}

Palabras-clave: Mapa mental. Aprendizaje cooperativo. Tecnologías de la información y la comunicación. Estrategia de aprendizaje. Educación superior.

\section{THE LEARNING OF MIND MAP IN GROUP THROUGH ICT IN THE INITIAL TEACHING TRAINING}

\begin{abstract}
The objective of this study focuses on the learning of the mind map as a study technique in future education professionals, through a cooperative learning dynamic, as well as Mindmanager 7 software for its construction. To reach this goal, a quantitative methodology based on a 22 -item ad hoc questionnaire was used. The results obtained show that the combination of the use of the information and communication technologies (ICT) and teamwork contributes positively in the learning of mind maps, as well as in the assessment of students in the study scope despite the fact that, in certain aspects, the students' stance is of indifference.
\end{abstract}

Keywords: Mind map. Cooperative learning. Information and communication technologies. Learning strategies. Higher education.

1.Universidad de Córdoba - Facultad de Ciencias de la Educación - Córdoba, España. E-mail: juan.manuel@uco.es

2.Universidad de Córdoba - Facultad de Ciencias de la Educación - Córdoba, España. E-mail: z52vegee@uco.es

3.Universidad de Córdoba - Facultad de Ciencias de la Educación - Córdoba, España. E-mail: lola.hidalgo@uco.es 


\title{
A APRENDIZAGEM DO MAPA MENTAL EM GRUPO ATRAVÉS DAS TIC NA FORMAÇÃO INICIAL DE PROFESSORES
}

\begin{abstract}
RESUMO: O objetivo deste estudo é aprender o mapa mental como técnica de estudo para futuros profissionais da educação por meio de uma dinâmica de aprendizado cooperativo, bem como o software Mindmanager 7 para sua construção. Para isso, adotamos um método quantitativo, utilizando um questionário criado ad hoc e composto por 22 itens. Os resultados obtidos mostram que a combinação do uso de tecnologias de informação e comunicação (TIC) e de trabalho em equipe contribuiu positivamente para a aprendizagem de mapas mentais, bem como para a avaliação desses no contexto do estudo, apesar de, em certos aspectos, a postura do aluno ser de indiferença.
\end{abstract}

Palavras-chave: Mapa mental. Aprendizagem cooperativa. Tecnologias de informação e comunicação. Estratégia de aprendizagem. Educação superior.

\section{Introducción}

$\mathrm{E}$

n la sociedad del siglo XXI emergen nuevas necesidades en cuanto a adquisición de competencias

y habilidades por parte de los docentes. La tradicional lección magistral ha pasado a la historia

en favor de nuevas dinámicas con una mayor participación del alumnado (MUÑOZ et al., 2016).

Estos nuevos planteamientos educativos han llevado a implementar técnicas y estrategias relacionadas con el enfoque cerebral y la construcción del conocimiento (NUÑEZ et al., 2019). En esta línea, se le da especial relevancia al concepto de "visualización de la información" (DÜRSTELER, 2002). Estos medios facilitadores de la visualización se denominan "organizadores gráficos".

Entre los organizadores gráficos cabe destacar el mapa mental, creado por Buzan durante la década de los 1970, el cual lo define como: "El mapa mental es una técnica mnemotécnica multidimensional que utiliza las funciones inherentes al cerebro para grabar en él, de manera más efectiva, los datos y la información” (BUZAN, 2004, p. 32).

El uso educativo de los mapas mentales tiene su fundamento en la teoría del aprendizaje constructivista en el que los conocimientos previos constituyen la base sobre la que sustentar la asimilación de nuevos conocimientos (NOVAK, 1991). Desde esta perspectiva, cobra especial relevancia este uso educativo como instrumento de representación del conocimiento (RÍOS et al., 2007). Desde el punto de vista del profesorado, suponen una herramienta adecuada para la estructuración de sus propios conocimientos y sus esquemas conceptuales a la hora de la creación de estos recursos didácticos (MAGLIONE; VARLOTTA, 2011). Asimismo, al ser utilizados en el aula, obligan a reflexionar sobre el propio conocimiento y permiten detectar las posibles deficiencias del proceso de aprendizaje (VÁZQUEZ-CANO; LÓPEZ-MENESES, 2016), así como recordar y comprender con mayor facilidad la información (LÓPEZ-MENESES; PÉREZ-PÉREZ, 2017; MUÑOZ et al., 2015).

En definitiva, su uso resulta de gran utilidad en la educación del alumnado, ya que éste aprende a construir sus propios modelos mentales individual o grupalmente. Igualmente, se ha constatado que los mapas mentales favorecen el aprendizaje cooperativo (STOKHOF et al., 2019). Estas características permiten considerarlos una actividad educativa necesaria en la formación de los futuros docentes (CAMPOS et al., 2009). 


\section{La Implementación de los Mapas Mentales a Través del Aprendizaje Cooperativo}

El aprendizaje cooperativo constituye un enfoque pedagógico en el que el alumnado se organiza en pequeños grupos con objeto de alcanzar un fin común. Los componentes del grupo deben aprender los contenidos, además de conseguir que el resto de compañeros/as también logre asimilarlos. Mediante este método, se pretende que todos los miembros del grupo se comprometan a alcanzar sus objetivos, lo que provoca una interdependencia positiva, a la vez que favorece la motivación y el aprendizaje (LÓPEZ; ACUÑA, 2011; PÉREZ et al., 2009; SANTOS et al., 2013). Como resultado, hay una evolución desde el trabajo individual y aislado hacia el trabajo en equipo, y desde una posición pasiva orientada exclusivamente a la recepción del conocimiento hacia una postura esencialmente activa. Esta técnica, por tanto, aprovecha todas las ventajas asociadas al aprendizaje entre iguales, aportando un mejor entendimiento de la materia explicada y mejorando las dotes para el consenso y la gestión de los conflictos entre las distintas opiniones (SERRANO et al., 2012). Por todo ello, las estrategias cooperativas de aprendizaje representan una solución adecuada para el docente, ya que, utilizadas de la manera correcta, permiten al alumnado apoyarse en sus compañeros/as durante el desarrollo de su aprendizaje y, de esta forma, adquirir y mejorar sus habilidades cognitivas, sociales y emocionales (TORREGO; NEGRO, 2012).

Consecuentemente, es en este contexto del aprendizaje cooperativo donde los mapas mentales están adquiriendo cada vez más una mayor importancia como instrumento de aprendizaje (MUÑOZ et al., 2016), ya que esta herramienta puede ser aplicada, por un lado, al aprendizaje cooperativo intra-grupo, es decir, mediante la elaboración y compartición de los mapas mentales entre los propios miembros de un grupo, basándose en ellos para explicar las materias asignadas; y, por otro lado, en un sentido más amplio, en el marco del aprendizaje cooperativo entre los distintos equipos, diseñando mapas para exponer unos a otros los contenidos de la asignatura (COBAS, 2013). En conclusión, además de potenciar las capacidades para el trabajo en equipo, se mejoran las dotes para hablar en público y se desarrolla la capacidad de síntesis del alumnado (GESTOSO; MORAES, 2013), ya que se debe resumir toda la información existente en un mapa mental y transmitir al resto del grupo o de la clase los aspectos más relevantes de la misma. Por último, también aumenta el rendimiento general en los estudiantes, propiciado por el intercambio de ideas entre los miembros del grupo, lo que facilita el procesamiento de la información y su reestructuración (ONTORIA et al., 2006).

\section{El software Mindmanager para la Creación de mapas mentales}

Las TIC abren todo un nuevo abanico de posibilidades para la construcción de mapas mentales como estrategias de aprendizaje (PEIRANO; DOMÍNGUEZ, 2008). En la actualidad existen distintos tipos de software de apoyo a la elaboración de este tipo de organizadores gráficos, que permiten centrarse en la forma de conceptualización del tema en cuestión y dejar de preocuparse por las habilidades personales para el dibujo y la representación. Mediante estas herramientas, se puede agregar o eliminar fácilmente elementos y relaciones, modificar su posición, añadir imágenes que contribuyan a clarificar el tema etc. (MUÑOZ, 2010).

Como se ha comentado anteriormente, la construcción del mapa mental de manera cooperativa implica un proceso de negociación e interacción del alumnado, por lo que el uso del software, como Mindmanager (https://www.mindjet.com/es), puede facilitar la creación del mapa mental por partes, de tal manera que posteriormente se puedan realizar modificaciones y, a su vez, construir estructuras gráficas mucho más complejas y completas (MUNOZ, 2010). Esto habilita la posibilidad de experimentar colaborativamente 
distintas formas de mapa, desarrollando el proceso de toma de decisiones, así como a creatividad e imaginación a través de la integración de imágenes, colores y formas de origen digital. Por tanto, este tipo de software posibilita combinar la construcción de los mapas mentales con una estrategia metodológica alineada con un aprendizaje cooperativo o colaborativo.

La elección de Mindmanager como programa para el desarrollo de esta dinámica de aprendizaje estuvo motivada por varias razones, como son:

- La adquisición de una licencia campus, por parte de la Universidad de Córdoba, que permite al alumnado de cualquier carrera universitaria acceder y utilizar dicha aplicación sin coste alguno;

- El software ofrece una gran cantidad de elementos para la configuración de los mapas mentales (imágenes, iconos, agrupamientos, relaciones...), así como para la organización del trabajo en grupo (alertas, notas, estado de una tarea...), algunos de los cuales no se encuentran disponibles en otras aplicaciones;

- Además, permite la incorporación de diferentes tipos de recursos, como vídeos o enlaces que enriquecen el mapa en formato digital, así como el modo presentación, empleado para la exposición final del trabajo en clase;

- Por último, y no por ello menos importante, conviene resaltar que el proceso de elaboración del mapa mental a través de Mindmanager es muy similar a otros programas gratuitos, como Xmind o Freemind, por lo que, una vez adquiridas las destrezas necesarias para su creación, el alumnado puede emplear cualquier otro software para continuar su trabajo en otro contexto no universitario.

Una de las limitaciones que nos encontramos en esta versión, es la imposibilidad de trabajar simultáneamente, varios componentes del grupo en un mismo mapa mental, limitación que se ha superado en las nuevas versiones del programa.

En cuanto a la dinámica de aprendizaje de dicho software, se divide en varias partes, que son:

- Creación del tema central, así como de las ideas principales, secundarias...;

- Una vez creada la estructura de ideas, se procede a la incorporación de los colores, asignando los más cálidos en el tema central e ideas principales, así como más fríos al resto;

- Posteriormente, se integran las imágenes a la estructura creada;

- Por último, se establecen las relaciones entre las diferentes ideas, si las hubiera.

En definitiva, el software Mindmanager permite la creación de mapas mentales de una manera fácil y rápida, reduciendo el tiempo invertido respecto a su elaboración manual, y permitiendo corregir posibles errores que resultaría imposible en formato papel.

\section{Objetivos}

El estudio que presentamos aborda varios objetivos que concretamos a continuación:

1. Describir las opiniones del alumnado de los Grados de Educación Infantil y Primaria, así como del Máster de Educación Inclusiva de la Universidad de Córdoba respecto a las aportaciones del mapa mental en el ámbito del estudio, así como las relacionadas con su desarrollo a través de dinámicas de aprendizaje cooperativo y su elaboración a través del programa Mindmanager 7; 
2. Analizar si existen diferencias en función del sexo, la edad y la titulación del alumnado;

3. Observar la existencia o no de correlaciones entre las diferentes dimensiones del cuestionario;

4. Establecer modelos para predecir las aportaciones del mapa mental en el ámbito del estudio en función de la dinámica de aprendizaje cooperativo llevada a cabo y del uso del programa Mindmanager 7 empleado para su elaboración.

\section{Metodología}

\section{Muestreo y participantes}

La selección de la muestra la realizamos utilizando el procedimiento de muestreo no probabilístico o de conveniencia (CUENCA; LOZANO, 2016) debido a que la metodología de aprendizaje desarrollada en el aula, así como la aplicación del cuestionario, solo se pudieron realizar a los grupos de estudiantes a los que los docentes de este estudio impartían clase durante el curso académico 2016-2017.

La muestra estuvo compuesta por un total de 409 sujetos: 236 estudiantes de $2^{\circ}$ del Grado de Educación Infantil (57,7\%), 136 pertenecientes al Grado de Educación Primaria (33,3\%) y 37 correspondientes al Máster de Educación Inclusiva (9\%). Respecto al sexo, un 77,2\% de la muestra estuvo compuesta de chicas $(n=342)$.

En cuanto a la relación entre la edad y el sexo del alumnado, el rango de esta estuvo comprendido entre 18 y mayores de 26 años. En este sentido, más de la mitad de los estudiantes (50,4\%) tenían entre 18-20 años, siendo un 85\% chicas y un 15\% chicos. El 28,6\% tenían entre 21-23 años, del cual el 78.6\% eran chicas y el $21,4 \%$ chicos. Por otra parte, un $11,7 \%$ tenían entre $24-26$ años, del cual el 87,5\% eran chicas y el $12,5 \%$ chicos. Finalmente, el 9,3\% tenían más de 26 años, del cual el $86,8 \%$ eran chicas y el $13,2 \%$ chicos.

\section{Instrumento de recogida de datos}

El instrumento utilizado para llevar a cabo este estudio se trata de un cuestionario elaborado ad hoc, denominado Cuestionario sobre las aportaciones del mapa mental en el ámbito del estudio, compuesto de 22 ítems. La validación y fiabilidad del instrumento han sido medidas y corroboradas mediante diferentes análisis estadísticos. En cuanto a la validez de constructo, en primer lugar hemos realizado un análisis factorial exploratorio, utilizando para la ejecución de esta prueba el método de Implementación óptima del Análisis Paralelo (AP) (TIMMERMAN; LORENZO-SEVA, 2011), junto con un proceso de extracción de factores comunes "máxima verosimilitud robusto" (MLR), considerando un procedimiento de rotación "Oblimin Ponderado" (LORENZO-SEVA, 2000), cuyo índice Kaiser-Meyer-Olkin (KMO) fue de 0,96, test de esfericidad de Barllett con $\mathrm{p}=0,000$ y análisis de residuales con RMSR $=0,0276$, considerado adecuado para el modelo (ALDAS; URIEL, 2017). Por otra parte, la varianza presenta una distribución no normal, medida a través de la prueba de Kolmogorov-Smirnov $(\mathrm{p}=0,022)$. El resultado de la extracción de los componentes principales refleja la existencia de 3 factores, donde la varianza total explicada es de 70,74\%, lo cual revela un equilibrio apropiado entre los componentes del instrumento, representativos del concepto teórico. 
Tabla 1. Matriz de componentes rotados en el análisis factorial exploratorio.

\begin{tabular}{lccc}
\hline Item & $\begin{array}{c}\text { Factor 1. } \\
\text { Aportaciones del mapa mental } \\
\text { en el ámbito del estudio }\end{array}$ & $\begin{array}{c}\text { Factor 2. } \\
\text { Contribuciones de la dinámica grupal } \\
\text { al aprendizaje del mapa mental }\end{array}$ & $\begin{array}{c}\text { Factor 3. } \\
\text { en la construcción del mapa mental }\end{array}$ \\
\hline 1 & 0,782 & & \\
\hline 2 & 0,817 & & \\
\hline 3 & 0,803 & & \\
\hline 4 & 0,815 & & \\
\hline 5 & 0,869 & & \\
\hline 6 & 0,739 & 0,453 & \\
\hline 7 & 0,556 & 0,667 & \\
\hline 8 & 0,607 & 0,821 & 0,663 \\
\hline 9 & 0,553 & 0,724 & \\
\hline 10 & & 0,718 & 0,607 \\
\hline 11 & & 0,814 & \\
\hline 12 & & 0,549 & \\
\hline 13 & & 0,735 & \\
\hline 14 & & & \\
\hline 15 & & & \\
\hline 16 & & & \\
\hline 17 & & & \\
\hline 18 & & & \\
\hline 19 & & & \\
\hline 21 & & & \\
\hline
\end{tabular}

Con la intención de contrastar y confirmar el modelo extraído a través del AFE, hemos realizado un AFC mediante el programa estadístico AMOS, en su versión 23. Este análisis permite explicar las correlaciones entre un conjunto de variables observadas a través de un conjunto reducido de variables latentes o factores (HERRERO, 2010). De este modo, será posible comprobar cómo cada variable está relacionada con su factor y si los factores están interrelacionados entre sí. Los resultados obtenidos del AFC, tomando como referencia los valores de la función de discrepancia (chi-cuadrado), de ajuste relativo, así como los basados en la descentralización (Error cuadrático medio de aproximación; RMSEA) mostraron unos índices de ajuste adecuados en función del modelo $(\chi 2=412,662$; gl. $=165$; NFI = 0,946; RFI = 0,924; IFI =0,967; TLI = 0,953; RMSEA = 0,064), lo que llevó a aceptarlo, teniendo como base los resultados del AFE y las dimensiones teóricas consideradas en el mismo (SCHUMACKER; LOMAX, 2004; BROWNE; CUDECK, 1993; BYRNE, 1994; HU; BENTLER, 1998; STIEGER, 2000).

La medición de la fiabilidad del instrumento se ha llevado a cabo a través del enfoque de consistencia interna (MERINO-SOTO, 2016), obteniendo un Alfa de Cronbach de 0.95, lo cual refleja una fiabilidad alta de los ítems expresados en el cuestionario. Finalmente, la matriz de componentes rotados expresa que existen 3 factores que tratan de aportar una explicación al constructo estudiado: 1. Aportaciones del mapa mental en el ámbito del estudio $(\alpha=0,93) ; 2$. Contribuciones de la dinámica grupal al aprendizaje del mapa mental $(\alpha=0,93) ; 3$. Valoración del software Mindmanager 7 en la construcción del mapa mental $(\alpha=0,84)$. 
A través de los 22 ítems medidos en escala Likert de 5 puntos ( 1 = totalmente en desacuerdo a 5 = totalmente de acuerdo), se preguntó a los estudiantes para conocer su opinión acerca de las aportaciones de los mapas mentales en el ámbito del estudio. Además, se solicitó al alumnado que respondiese a una serie de preguntas directas que hacían alusión a sus datos personales (sexo y edad) y académicos (Titulación).

\section{Procedimiento}

El instrumento fue facilitado a los estudiantes al concluir la dinámica basada en el aprendizaje del mapa mental como técnica de estudio, dedicando 15 minutos para su cumplimentación. Los investigadores del estudio fueron los responsables tanto de llevar a cabo dicha metodología como de recoger los datos en todas las titulaciones.

\section{Análisis de Datos}

Los análisis que hemos llevado a cabo en este estudio y mediante los cuales hemos pretendido dar respuesta a los objetivos planteados son los siguientes:

- En primer lugar, hemos realizado un análisis descriptivo de las 22 variables que componían el cuestionario a través de los estadísticos de medida central (media) y de medida de dispersión (desviación típica);

- En segundo lugar, hemos llevado a cabo un análisis descriptivo de las 3 dimensiones del cuestionario, calculando la distribución de frecuencias, los estadísticos de medida central y las medidas de dispersión;

- En tercer lugar, realizamos diferentes análisis de varianza para comprobar si existía relación entre las dimensiones del cuestionario y las variables independientes: sexo, edad y titulación. Para ello, se llevaron a cabo las pruebas de U de Mann Whitney y K de Kruskal Wallis, utilizando el paquete estadístico SPSS 23;

- En cuarto lugar, procedimos a comprobar la relación entre las dimensiones que componían el cuestionario mediante correlaciones bivariadas;

- Por último, realizamos regresiones lineales múltiples para predecir las aportaciones del mapa mental en el ámbito de estudio en función de la dinámica de aprendizaje cooperativo llevada a cabo y del uso del programa Mindmanager 7 empleado para su elaboración.

\section{Resultado}

En primer lugar, se presentan los resultados descriptivos de los 22 ítems que componen el cuestionario utilizado en el presente trabajo de investigación. En la tabla 2 podemos observar las medias y desviaciones típicas de los ítems que componen el cuestionario sobre las aportaciones del mapa mental en el ámbito del estudio. 
Tabla 2. Distribución de frecuencias de los ítems del cuestionario sobre las aportaciones del mapa mental en el ámbito del estudio.

\begin{tabular}{|c|c|c|c|}
\hline Factores & Ítem & M & DT \\
\hline \multirow{9}{*}{$\begin{array}{l}\text { Factor } 1 . \\
\text { Aportaciones del } \\
\text { mapa mental en el } \\
\text { ámbito del estudio }\end{array}$} & 1. Me facilitó la comprensión de los documentos trabajados & 3,69 & 1,02 \\
\hline & 2. Me facilitó la síntesis de la información & 3,77 & 0,94 \\
\hline & 3. Me facilitó la organización de los contenidos & 3,87 & 0,91 \\
\hline & 4. Me facilitó el recuerdo de los contenidos & 3,89 & 0,88 \\
\hline & 5. Me facilitó el estudio de los documentos trabajados & 3,81 & 0,93 \\
\hline & 6. Potenció la eficacia en el aprendizaje & 3,77 & 0,93 \\
\hline & 7. El proceso de elaboración facilita la obtención de las ideas principales & 4,05 & 0,94 \\
\hline & 8. La estrategia incrementa la rapidez en el estudio & 3,74 & 0,99 \\
\hline & 9. La técnica aumenta la eficacia en el estudio/aprendizaje & 3,85 & 0,96 \\
\hline \multirow{8}{*}{$\begin{array}{l}\text { Factor } 2 . \\
\text { Contribuciones } \\
\text { de la dinámica } \\
\text { grupal al } \\
\text { aprendizaje del } \\
\text { mapa mental }\end{array}$} & 10. La dinámica de grupo facilita la compresión de los temas trabajados & 4,00 & 0,86 \\
\hline & 11. La dinámica de grupo facilita la elaboración del mapa & 3,87 & 0,90 \\
\hline & 12. La dinámica de grupo repercute en la mejor resolución de dificultades & 3,91 & 0,95 \\
\hline & 13. La dinámica de grupo facilita el desarrollo de debates & 3,86 & 0,92 \\
\hline & 14. El mapa mental fomenta el rendimiento en el grupo & 3,75 & 0,93 \\
\hline & 15. El trabajo en grupo con mapas mentales favorece el clima de sus componentes & 3,71 & 0,95 \\
\hline & 16. La dinámica seguida potencia la aportación de ideas en el grupo & 3,85 & 0,88 \\
\hline & 17. La dinámica seguida mejora las relaciones personales en el grupo & 3,61 & 1,00 \\
\hline \multirow{5}{*}{$\begin{array}{l}\text { Factor } 3 . \\
\text { Valoración } \\
\text { del software } \\
\text { Mindmanager } 7 \\
\text { en la construcción } \\
\text { del mapa mental }\end{array}$} & 18. Mindmanager facilita el establecimiento de relaciones entre ideas & 3,94 & 0,92 \\
\hline & 19. El software facilita la elaboración del mapa individual & 3,81 & 0,96 \\
\hline & 20. El entorno de la aplicación es intuitivo y facilita su uso & 3,51 & 1,01 \\
\hline & 21. El programa usado facilita la elaboración del mapa consensuado & 3,63 & 0,98 \\
\hline & 22. El software usado tiene aplicabilidad en diferentes entornos educativos & 3,55 & 1,01 \\
\hline
\end{tabular}

A continuación, mostramos los resultados agrupados en función de las 3 dimensiones de las que consta el cuestionario. En este sentido, exponemos los datos descriptivos de cada una de las dimensiones, así como la existencia de diferencias estadísticamente significativas, atendiendo a las variables independientes del estudio (sexo, edad y titulación). Finalmente, se presenta la relación existente entre las dimensiones que componen el cuestionario, así como el establecimiento de un modelo predictor de las opiniones del alumnado respecto a las aportaciones del mapa mental en el ámbito de estudio en función de la dinámica de aprendizaje cooperativo llevada a cabo y del uso del programa Mindmanager 7 empleado para su elaboración.

\section{Aportaciones del Mapa Mental en el Ámbito del Estudio}

En esta dimensión, agrupamos 9 ítems que hacían alusión a las opiniones del alumnado en cuanto a las aportaciones del mapa mental en el ámbito del estudio. Concretamente, se alude a sus posibilidades en cuanto a la comprensión, organización, síntesis y recuerdo de los contenidos trabajados en clase, así como la rapidez y eficacia en el estudio.

En los resultados obtenidos en esta dimensión, se observó que el alumnado manifiesta una opinión de indiferencia respecto a las aportaciones del mapa mental en el ámbito de estudio $(\mu=3,83 ; \sigma=0,77)$, aunque el valor está próximo a parcialmente de acuerdo. 
En cuanto a las diferencias que el sexo establecía en relación a las opiniones del alumnado en relación a las aportaciones de los mapas mentales en el ámbito del estudio, la prueba $U$ de Mann Whitney para muestras independientes señaló que existían diferencias estadísticamente significativas en función del sexo de los estudiantes $[U=9164,500,(67,342), \mathrm{z}=-2,595, \mathrm{p}<0,01]$. En este sentido, las chicas presentaban unos rangos promedios superiores a los de los chicos en cuanto a las aportaciones del mapa mental en el ámbito del estudio.

Para determinar la influencia de la edad y la titulación sobre los resultados, hemos realizado comparaciones múltiples de media a través de análisis $K$ de Kruskal Wallis. Los resultados obtenidos mostraron que no había diferencias estadísticamente significativas entre la edad y las opiniones de los estudiantes respecto a las aportaciones del mapa mental en el ámbito del estudio.

En cuanto a la relación de la titulación del alumnado y sus opiniones, los análisis mostraron resultados similares a los anteriores, es decir no existían diferencias estadísticamente significativas entre la titulación y el sentir de los estudiantes respecto a las aportaciones del mapa mental en el ámbito del estudio.

\section{Contribuciones de la Dinámica Grupal al Aprendizaje del Mapa Mental}

Esta dimensión está compuesta por 8 ítems en los que se recogen las opiniones de los estudiantes en relación a la contribución de la dinámica grupal al aprendizaje del mapa mental como técnica de estudio. Específicamente, se hace referencia a aspectos relacionados con la aportación de ideas de los diferentes miembros del grupo, posibilidades de debate, facilidad en la comprensión de los contenidos trabajados y en la elaboración del mapa mental, resolución de dificultades en el aprendizaje de este, así como a la mejora de las relaciones entre los integrantes del mismo.

En los resultados obtenidos en esta dimensión, observamos que el alumnado manifiesta una valoración de indiferencia respecto a las aportaciones de la dinámica grupal en el aprendizaje del mapa mental $(\mu=3,82$; $\sigma=0,76)$, aunque, al igual que en la dimensión anterior, el valor está próximo a parcialmente de acuerdo.

Por otra parte, la prueba $U$ de Mann Whitney, realizada para identificar posibles diferencias en relación al sexo del alumnado en cuanto a las aportaciones de la dinámica grupal en el aprendizaje del mapa mental, indicó que existían diferencias estadísticamente significativas $[U=9680,500,(67,342), z=-2,013$, $\mathrm{p}<0,05]$. En este sentido, las chicas presentaban unos rangos promedios superiores a los de los chicos en cuanto a las aportaciones de la dinámica grupal en el aprendizaje del mapa mental.

Por otra parte, los análisis de varianza realizados para constatar la existencia de diferencias significativas entre la edad y la titulación en relación a las aportaciones de la dinámica grupal en el aprendizaje del mapa mental mostraron que no existían diferencias estadísticamente significativas en relación a la edad. En cuanto a la titulación de los estudiantes respecto a las aportaciones de la dinámica grupal, los análisis tampoco arrojaron diferencias significativas.

\section{Valoración del Software Mindmanager 7 en la Construcción del Mapa Mental}

Esta dimensión está compuesta por 5 ítems en los que se valora las posibilidades del programa Mindmanager 7 en la construcción del mapa mental. Concretamente, se hace referencia al entorno de la aplicación, sus potencialidades a la hora de crear el mapa mental tanto de manera individual como grupal, así como las posibilidades para aplicarlo en las diferentes etapas educativas. 
En los resultados obtenidos en esta dimensión, observamos que el alumnado manifiesta una valoración de indiferencia en relación a la dimensión "Valoración del software Mindmanager 7 en la construcción del mapa mental” ( $\mu=3,69 ; \sigma=0,76)$ aunque, al igual que en los casos anteriores, el valor está próximo a parcialmente de acuerdo.

La prueba $U$ de Mann Whitney, realizada para identificar posibles diferencias en relación al sexo del alumnado en cuanto a la valoración del programa Mindmanager 7 para la creación del mapa mental indicó que no existían diferencias estadísticamente significativas.

Por otra parte, los análisis de varianza realizados a través de la prueba $K$ de Kruskal Wallis para constatar la existencia de diferencias significativas entre la edad y la titulación del alumnado en relación a las valoraciones de dicho software para la creación del mapa mental, mostraron que tampoco existían diferencias estadísticamente significativas en ninguno de los dos casos.

\section{Estudio Correlacional}

En este apartado, abordaremos el estudio correlacional entre las 3 dimensiones del cuestionario. Los datos resultantes de la aplicación de la prueba de correlación de Spearman, realizada para comprobar la relación entre las 3 dimensiones de la escala pueden verse en la Tabla 3.

Tabla 3. Resultados de las correlaciones bivariadas de las dimensiones del cuestionario sobre las aportaciones del mapa mental en el ámbito del estudio.

\begin{tabular}{|c|c|c|c|c|c|}
\hline & & & Estudio & Grupo & Mindmanager \\
\hline \multirow{9}{*}{$\begin{array}{c}\text { Rho de } \\
\text { Spearman }\end{array}$} & \multirow{3}{*}{ Estudio } & Coeficiente de correlación & 1,000 & $0,672^{\star \star}$ & $0,691^{\star *}$ \\
\hline & & Sig. (bilateral) & & 0,000 & 0,000 \\
\hline & & $\mathrm{N}$ & 409 & 409 & 409 \\
\hline & \multirow{3}{*}{ Grupo } & Coeficiente de correlación & $0,672^{\star \star}$ & 1,000 & $0,747^{\star *}$ \\
\hline & & Sig. (bilateral) &, 000 & & 0,000 \\
\hline & & $\mathrm{N}$ & 409 & 409 & 409 \\
\hline & \multirow{3}{*}{ Mindmanager } & Coeficiente de correlación & $0,691^{\star *}$ & $0,747^{\star *}$ & 1,000 \\
\hline & & Sig. (bilateral) &, 000 & 0,000 & \\
\hline & & $\mathrm{N}$ & 409 & 409 & 409 \\
\hline
\end{tabular}

** La correlación es significativa en el nivel 0,01 (bilateral).

En función de los datos obtenidos, podemos afirmar que existe relación entre la dimensión 1 con la dimensión 2 y con la dimensión 3, respectivamente $R=0,672$ y $p=0,000 ; R=0,691$ y $p=0,000$, dado que existe nivel de significatividad bilateral al n.s $=0,01$. La relación entre las mismas es alta, tal y como señalan Mateo (2004) y Pérez et al. (2009).

Por último, podemos afirmar que también existe relación entre la dimensión 2 y la 3 , ya que $\mathrm{R}=0,747$ y $\mathrm{p}=0,000$, dado que existe nivel de significatividad bilateral al $\mathrm{n} . \mathrm{s}=0,01$. La relación entre las mismas es igualmente alta, tal y como señalan los autores anteriormente mencionados. 


\section{Modelos Explicativos de las Opiniones del Alumnado Respecto a las Aportaciones del Mapa Mental en Función de la Dinámica llevada a Cabo y Del uso del Programa Mindmanager 7}

En este punto tratamos de explicar la variable "aportaciones del mapa mental en el ámbito del estudio" en función de las medidas de las dimensiones "contribuciones de la dinámica grupal al aprendizaje del mapa mental" y "valoración del software Mindmanager 7 en la construcción del mapa mental” a través de regresiones lineales múltiples (PARDO; RUIZ, 2002), utilizando el método “por pasos”, con el fin de observar las variables predictoras y su relación con la variable criterio.

Tabla 4. Coeficientes de la recta de regresión para la variable dependiente "aportaciones del mapa mental en el ámbito del estudio" en función de las medidas de las dimensiones "contribuciones de la dinámica grupal al aprendizaje del mapa mental" y "valoración del software Mindmanager 7 en la construcción del mapa mental".

\begin{tabular}{|c|c|c|c|c|c|c|c|c|}
\hline & \multirow{2}{*}{$\begin{array}{c}\text { Modelo } \\
\text { B }\end{array}$} & \multicolumn{2}{|c|}{$\begin{array}{l}\text { Coeficientes no } \\
\text { estandarizados }\end{array}$} & \multirow{2}{*}{$\begin{array}{l}\text { Coeficientes } \\
\text { estandarizados }\end{array}$} & \multirow{2}{*}{$\mathrm{t}$} & \multirow{2}{*}{ Sig. } & \multicolumn{2}{|c|}{$\begin{array}{l}\text { Estadísticas de } \\
\text { colinealidad }\end{array}$} \\
\hline & & Error estándar & Beta & & & & Tolerancia & VIF \\
\hline \multirow{2}{*}{1} & Constante & 1,178 & 0,133 & & 8,839 & 0,000 & & \\
\hline & Mindmanager & 0,718 & 0,035 & 0,710 & 20,325 & 0,000 & 1,000 & 1,000 \\
\hline \multirow{3}{*}{2} & Constante & 0,895 & 0,138 & & 6,479 & 0,000 & & \\
\hline & Mindmanager & 0,470 & 0,056 & 0,464 & 8,413 & 0,000 & 0,372 & 2,685 \\
\hline & Grupo & 0,314 & 0,056 & 0,310 & 5,614 & 0,000 & 0,372 & 2,685 \\
\hline
\end{tabular}

${ }^{a}$ Variable dependiente: Aportaciones del mapa mental al ámbito del estudio.

Los resultados obtenidos, tal y como podemos ver en la tabla 4, nos permiten observar que las dimensiones "contribuciones de la dinámica grupal al aprendizaje del mapa mental" y "valoración del software Mindmanager 7 en la construcción del mapa mental” constituyen factores predictores de la dimensión "Aportaciones del mapa mental en el ámbito del estudio", ya que $\beta=0,464, \mathrm{t}(464)=8,413, \mathrm{p}<0.01 ; \mathrm{y} \beta=0,310$, $\mathrm{t}(310)=5,614, \mathrm{p}<.01$, respectivamente. Por tanto, es estadísticamente significativa, por lo que aceptamos la hipótesis de relación lineal entre las contribuciones de la dinámica grupal al aprendizaje del mapa mental y la valoración del software Mindmanager 7 empleado en la construcción del mismo, en relación con las opiniones del alumnado en cuanto a las aportaciones de esta técnica en el ámbito del estudio. En este sentido, el valor de $\mathrm{R}^{2}$ ajustado fue de 0,537 , indicando que un 53,7\% de la variabilidad de la dimensión "Aportaciones del mapa mental en el ámbito del estudio" es explicada por las dos variables citadas anteriormente.

\section{Conclusiones y Discusión}

En este apartado se recogen las principales conclusiones a las que se ha llegado partir de los resultados de la presente investigación. La exposición de dichas conclusiones se realiza siguiendo el orden de los objetivos planteados en el estudio.

El análisis descriptivo de las aportaciones del mapa mental en el ámbito de estudio indicó que el alumnado percibe que el proceso de elaboración del mapa mental facilita la obtención de ideas principales, sin embargo se mostraron indiferentes o tenían sus dudas con respecto a aspectos tales como si el uso del mapa mental les facilitaba la comprensión de los documentos trabajados, la síntesis de la información o la 
potenciación de la eficacia en el aprendizaje, así como el incremento de la rapidez en el estudio. En cuanto a las contribuciones de la dinámica grupal al aprendizaje del mapa mental, los estudiantes señalaron que la dinámica de grupo facilita la comprensión de los temas trabajados y repercute en una mejor resolución de las dificultades que se presentan, pero tenían sus dudas o se manifestaron indiferentes en cuanto a que la dinámica grupal mejora las relaciones personales en el grupo. En lo referente a la valoración del software Mindmanager 7 en la construcción del mapa mental, el alumnado indicó que la utilización del software les facilitaba el establecimiento de relaciones entre ideas, pero por el contrario se mostraron indiferentes o tenían dudas en cuanto a si el entorno de la aplicación era intuitivo y facilitaba su uso, así como si tenía aplicabilidad en otros entornos educativos. En este sentido, los resultados coinciden con los obtenidos en estudios previos (MUÑOZ et al., 2014; MUÑOZ et al., 2016), en los que se señala la importancia de la percepción y valoración que los futuros docentes tienen de las aportaciones del uso de los mapas mentales en el ámbito de estudio en general, y en las dinámicas de clase en particular.

Seguidamente, se comprobó si existían diferencias en las dimensiones del cuestionario en relación con el sexo, la edad y la titulación que estaban cursando los y las estudiantes. En este caso, los datos resultantes han indicado que la opinión del alumnado en relación al sexo únicamente presentaba diferencias significativas en relación a las aportaciones del mapa mental en el ámbito educativo. En concreto, las chicas manifestaron en mayor proporción que los chicos una percepción personal más positiva de las aportaciones del mapa mental en el ámbito de estudio. Sin embargo, no se encontraron diferencias entre unos y otras en la valoración de las contribuciones de la dinámica grupal al aprendizaje del mapa mental, ni en la valoración del software Mindmanager 7 en la construcción de este. Por su parte, tanto la edad como la titulación no mostraron diferencias significativas en ninguna de las tres dimensiones estudiadas, es decir, la edad y la titulación no parecen ser un criterio de diferenciación en la percepción y valoración que el alumnado tiene de las aportaciones del mapa mental en el ámbito de estudio. Estos datos concuerdan parcialmente con los obtenidos en estudio anteriores (MUÑOZ et al., 2014; MUÑOZ et al., 2016). Al respecto, parece ser que no existe un acuerdo generalizado en los estudios realizados hasta la presente sobre el carácter que imprime el sexo, la edad y la titulación en relación a la valoración del mapa mental en el estudio, en la dinámica grupal y en la valoración de herramientas informáticas para su elaboración.

En lo relativo a la relación de las dimensiones del cuestionario, existe una alta correlación en todas ellas. No obstante, la correlación más alta se encontró entre las contribuciones de la dinámica grupal al aprendizaje del mapa mental y la valoración del software Mindmanager 7 en la construcción del mismo. Estos datos no se han podido contrastar con estudios anteriores, ya que no se han encontrado investigaciones que hayan medido la relación entre estas dimensiones. No obstante, esta alta correlación entre las dimensiones va en línea con la idea de que los recursos TIC favorecen la implantación de métodos activos de enseñanza (PONTES; SERRANO, 2008), dentro de los cuales se insertan las dinámicas grupales y por tanto, el aprendizaje colaborativo (PONTES et al., 2011). Por tanto, podría decirse que el uso de herramientas y software, como el Mindmanager 7, mejora los procesos de enseñanza aprendizaje, ya que permite la creación y visualización de los conocimientos adquiridos por el alumnado favoreciendo la creatividad, mientras se trabaja colaborativamente. Todo ello pone de manifiesto que la combinación del uso de las TIC y el trabajo en equipo en la elaboración de los mapas mentales contribuye a la mejora de la calidad de la educación y a una percepción más positiva del alumnado (DE ARRIBA, 2008).

En último lugar, mencionar que las aportaciones del mapa mental en el ámbito educativo 
constituyen un factor predictor de las contribuciones de la dinámica grupal al aprendizaje del mapa mental y de la valoración del software Mindmanager 7 en la construcción del mismo.

En definitiva, en la sociedad del siglo XXI resulta indispensable proporcionar una formación adecuada a los futuros docentes que les permita diseñar y llevar a cabo dinámicas de clase que respondan a los diferentes estilos, ritmos, intereses y motivaciones del alumnado de este siglo, constituyendo las tecnologías de la información y comunicación, así como los mapas mentales herramientas a tener en cuenta en los procesos de enseñanza/aprendizaje (CABERO; ROMERO, 2007; MUÑOZ et al., 2014; MUÑOZ et al., 2016; PRIEGUE; CRESPO, 2012).

\section{Contribuición de los autores}

Conceptualización de la investigación, Ariza MDH; Revisión de la literatura: Ariza MDH; Instrumentos de recolección y análisis de dados, González JMM; Discusión, Gea EMV; Escritura, Gea EMV; Corrección de estilo, González JMM.

\section{Referencias}

ALDAS, J.; URIEL, E. Análisis multivariante aplicado con R. Madrid: Paraninfo, 2017.

BROWNE, M. W.; CUDECK, R. Alternative ways of assessing model fit. In: BOLLEN, K. A.; LONG, J. S. (eds.), Testing structural equation models, p. 136-162, 1993. https://doi. org/10.1177/0049124192021002005

BUZAN, T. Tu mente en forma. Barcelona: Urano, 2004.

BYRNE, B. M. Structural equation modeling with EQS and EQS/Windows. Thousand Oaks: Sage Publications, 1994.

CABERO, J.; ROMERO, R. Diseño y producción de TIC para la formación. Nuevas tecnologías de la información y comunicación. Barcelona: Editorial UOC, 2007.

CAMPOS, M. C.; GARRIDO, M. E.; CASTAÑEDA, C. Técnicas para promover el aprendizaje significativo en educación física: Los Cmap-Tools. Retos: Nuevas tendencias en educación física, deporte y recreación, v. 16, p. 58-62, 2009.

COBAS, M. E. Aprendizaje cooperativo y mapas conceptuales. Valencia: Repoexpres, 2013.

CUENCA, A.; LOZANO, S. La enseñanza de la investigación. Diálogo entre la teoría y el oficio del investigador en Trabajo Social. La Plata: Editorial de la Universidad Nacional de La Plata (EDULP), 2016.

DE ARRIBA, J. A. Aprendiendo a resolver casos reales mediante la utilización de herramientas informáticas de aprendizaje y colaboración. Estudio experimental en un contexto de formación universitario. Revista de Universidad y Sociedad del Conocimiento, v. 5, n. 2, p. 36-49, 2008.

DÜRSTELER, J. C. Visualización de la información. Una visita guiada. Barcelona: Gestión 2000. com, 2002. 
GESTOSO, A. P.; MORAES, R. M. Aprendizagem da docência em grupo colaborativo: Histórias infantis e matemática. Educação e Pesquisa, v. 39, n. 4, p. 859-874, 2013. https://doi.org/10.1590/ S1517-97022013005000020

HERRERO, J. El Análisis Factorial Confirmatorio en el estudio de la estructura y estabilidad de los instrumentos de evaluación: un ejemplo con el Cuestionario de Autoestima CA-14. Intervención Psicosocial, v. 19, n. 3, p. 289-300, 2010.

HU, L. T.; BENTLER, P. M. Evaluating model fit. In: HOYLE, R. H. (ed.). Structural equation modeling: Concepts, issues, and applications, p. 76-99, 1995.

LÓPEZ, G.; ACUÑA, S. Aprendizaje cooperativo en el aula. Inventio, la génesis de la cultura universitaria en Morelos, v. 14, p. 28-37, 2011.

LÓPEZ-MENESES, E.; PÉREZ-PÉREZ, I. Ámbitos de intervención del educador/a y trabajador/a social: La percepción del estudiantado a través de los mapas conceptuales interactivos (MCI). El Guiniguada. Revista de investigaciones y experiencias en ciencias de la educación, v. 26, p. 104-119, 2017.

LORENZO-SEVA, U. The weighted oblimin rotation. Psychometrika, v. 65, p. 301-318, 2000. https:// doi.org/10.1007/BF02296148

MAGLiOnE, C.; VARLOTTA, N. Mapas conceptuales digitales. Buenos Aires: Educar S. E., 2011.

MATEO, J. La investigación 'ex post-facto'. In: BISQUERRA, R. (coord.). Metodología de investigación educativa, p. 196-230, 2004.

MERINO-SOTO, C. Diferencias entre coeficientes alfa de Cronbach, con muestras y partes pequeñas: Un programa VB. Anales de Psicología, v. 32, n. 2, p. 587-588, 2016. https://doi.org/10.6018/ analesps.32.2.203841

MUÑOZ, J. M. Los mapas mentales como técnica para integrar y potenciar el aprendizaje holístico en la formación inicial de maestros/as. 2010. Tesis (Doctorado en Ciencias de la Educación) Facultad de Ciencias de la Educación. Universidad de Córdoba, 2010.

MUÑOZ, J. M.; ARIZA, C.; SAMPEDRO, B. E. La aplicación de los mapas mentales en Educación Primaria. International Journal of Educational Research and Innovation (IJERI), v. 4, p. 70-89, 2015.

MUÑOZ, J. M.; HINOJOSA, E. F.; VEGA-GEA, E. Opiniones de estudiantes universitarios acerca de la utilización de mapas mentales en dinámicas de aprendizaje cooperativo. Estudio comparativo entre la Universidad de Córdoba y La Sapienza. Perfiles Educativos, v. 38, n. 153, p. 136-151, 2016.

MUÑOZ, J. M.; ONTORIA, A.; MOLINA, A. El mapa mental, un organizador grafico como estrategia didáctica para la construcción del conocimiento. Magis: Revista Internacional de Investigación en Educación, v. 3, n. 6, p. 343-361, 2011. doi: https://doi.org/10.11144/Javeriana.m3-6.mmog

MUÑOZ, J. M.; SERRANO, R.; MARÍN, V. El aprendizaje colaborativo y su desarrollo a través de mapas mentales. Una innovación educativa en la formación inicial docente. Educatio Siglo XXI, v. 32, n. 1, p. 193-212, 2014. https://doi.org/10.6018/j/194151 
NOVAK, J. D. Ayudar a los alumnos a aprender cómo aprender. La opinión de un profesor-investigador. Enseñanza de las Ciencias, v. 9, n. 3, p. 215-227, 1991.

NUÑEZ, L.; NOVOA, P.; MAJO, H.; SALVATIERRA, A. Mental maps as a strategy in the development of successful intelligence in high school students. Propósitos y Representaciones, v. 7, n. 1, p. 59-82, 2019.

OKADA, A. Cartografia cognitiva. Mapas do conhecimento para pesquisa, aprendizagem e formação docente. Cuiabá: KCM Editora, 2008.

ONTORIA, A.; GÓMEZ, J. P.; MOLINA, A.; DE LUQUE, A. Aprender con mapas mentales. Madrid: Narcea, 2006.

PARDO, A.; RUIZ, M. SPSS 11. Guía para el análisis de datos. Madrid: McGraw-Hill, 2002.

PEIRANO, C.; DOMÍNGUEZ, M. P. Competencia en TIC: El mayor desafío para la evaluación y el entrenamiento docente en Chile. Revista Iberoamericana de Evaluación Educativa, v. 1, n. 2, p. 106-124, 2008.

PÉREZ, R.; GARCÍA, J. L.; GIL, J. A.; GALÁN, A. Estadística aplicada a la Educación. Madrid: Pearson Prentice Hall, 2009.

PÉREZ, A. M.; LÓPEZ, M. P.; POVEDA, P. Aprendizaje cooperativo y formación del profesor: Un estudio bibliométrico (1997-2008). Anales de Documentación. Revista de Bibliotecomonomía y Documentación, v. 12, p. 209-220, 2009.

PONTES, A.; SERRANO, R. Mapas conceptuales y tecnología educativa. In: PONTES. A. (coord.). Aspectos Generales de la Formación Psicopedagógica del Profesorado de Educación Secundaria, p. 309-331, 2008.

PONTES, A.; SERRANO, R.; MUÑOZ, J. M.; LÓPEZ, I. Innovación educativa sobre aprendizaje colaborativo con CMAPTOOLS en la formación inicial docente. Revista Iberoamericana de Evaluación Educativa, v. 4, n. 2, p. 137-152, 2011.

PRIEGUE, D.; CRESPO, J. M. El potencial pedagógico de la tecnología: Desarrollar competencias y favorecer la autonomía y la responsabilidad en el alumnado. Educación en el Conocimiento de la Sociedad, v. 13, n. 2, p. 404-423, 2012. http://dx.doi.org/10.14201/eks.9016

RÍOS, L. R.; LÓPEZ, E.; LESCANO, M.; HERNÁNDEZ, A.; GARCÍA, A. Los mapas conceptuales, las TIC y el e-learning. Revista Iberoamericana de Educación, v. 43, n. 1, p. 1-8, 2007.

SANTOS, M. A.; LORENZO, M.; PRIEGUE, D. Conectando a los profesores para el desarrollo de la interculturalidad. Educación XX1, v. 26, n. 1, p. 63-84, 2013. https://doi.org/10.5944/educxx1

SCHUMACKER, R. E.; LOMAX, R. G. A beginner's guide to structural equation modeling. 2. ed. Mahwah: Lawrence Erlbaum Associates, 2004.

SERRANO, R.; MUÑOZ, J. M.; LÓPEZ, I. Valoración por los estudiantes de la licenciatura en psicopedagogía, de una experiencia innovadora basada en estrategias de aprendizaje colaborativo y 
el uso del Courselab. Teoría de la Educación: Educación y cultura en la sociedad de la información, v. 13, n. 1, p. 437-458, 2012. http://doi.org/10.14201/eks.8820

STEIGER, J. H. Point estimation, hypothesis testing and interval estimation using the RMSEA: Some comments and a reply to Hayduk and Glaser. Structural Equation Modeling, v. 7, p. 149-162, 2000. https://doi.org/10.1207/S15328007SEM0702_1

STOKHOF, H.; DE VRIES, B.; BASTIAENS, T.; MARTENS, R. Mind map our way into effective student questioning: A principle-based scenario. Research in Science Education, v. 49, n. 2, p. 347-369, 2019. https://doi.org/10.1007/s11165-017-9625-3

TIMMERMAN, M. E.; LORENZO-SEVA, U. Dimensionality assessment of ordered polytomous items with parallel analysis. Psychological Methods, v. 16, p. 209-220, 2011. https://doi.org/10.1037/ a0023353

TORREGO, J. C.; NEGRO, A. Aprendizaje cooperativo en las aulas. Madrid: Alianza Editorial, 2012. VÁZQUEZ-CANO, E.; LÓPEZ-MENESES, E. J.. Experiencia didáctica con mapas conceptuales interactivos con estudiantes universitarios sobre las áreas de intervención sociolaboral del educador social. Aula de Encuentro, v. 18, n. 1, p. 5-23, 2016.

\section{Sobre los Autores}

Juan Manuel Muñoz González es licenciado en Psicopedagogía y Doctor por la Universidad de Córdoba en Educación, centra su línea de investigación en la tecnología educativa, concretamente en el diseño de recursos didácticos digitales, así como estrategias de aprendizaje holístico, como los mapas mentales.

Esther Maria Vega Gea es licenciada en Psicopedagogía y Doctora por la Universidad de Córdoba en Educación, centra su línea de investigación en convivencia y prevención de la violencia escolar y juvenil, tanto de fenómenos cara a cara (bullying y dating violence) como en aquellos en los que se hace uso de las Redes sociales y TIC (cyberbullying, ciberdating violence y sexting entre otras).

María Dolores Hidalgo Ariza es licenciada en Psicopedagogía y Doctora por la Universidad de Córdoba en Educación, centra su línea de investigación en desigualdades de género en educación, concretamente en el estudio de roles y estereotipos de género, así como en el análisis de la brecha digital de género e investigación de las Redes Sociales con perspectiva de Género.

Recibido: 08 Feb 2019

Aceptado: 14 Feb 2020 